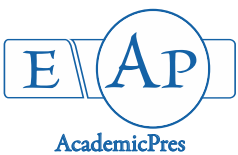

Thaner Dos Santos H et al. (2020)

Notulae Scientia Biologicae 12(1):42-56

DOI: $10.15835 / \mathrm{nsb} 12110588$

Research Article

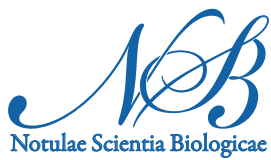

\title{
Effects of irrigation management and seasonal stages on essential oil content and biomass of Origanum vulgare $\mathrm{L}$.
}

\section{Hugo THANER DOS SANTOS ${ }^{1 *}$, Renata ALCARDE SERMARINI², Maria A. MORENO-PIZANI ${ }^{3}$, Patricia A. ALVES MARQUES ${ }^{4}$}

\author{
${ }^{1}$ Federal University of Viçosa, Agricultural Engineering Department, Av. P.H. Rolfs, Campus UFV, Viçosa-MG, \\ Brazil; hthaner@gmail.com (*corresponding author) \\ ${ }^{2}$ University of São Paulo, Department of Math, Chemistry and Statistics, Av. Pádua Dias, 11, ESALQ-LCE, Piracicaba-SP, \\ Brazil; ralcarde@usp.br \\ ${ }^{3}$ Programa de Educação Continuada em Economia e Gestão de Empresas (PECEGE), Rua Alexandre Herculano, 120-T6, Piracicaba- \\ SP,Brazil; morenom76@gmail.com \\ ${ }^{4}$ University of São Paulo, Biosystems Engineering Department, Av. Pádua Dias, 11, ESALQ-LEB, Piracicaba-SP, \\ Brazil;paamarques@usp.br
}

\begin{abstract}
The responses of oregano plants to water limitation from soil and seasonal phenological cycle are not fully understood yet. The aim of the present research was to help understanding the production of oregano essential oil and biomass facing soil water deficit, which was studied in different seasons. Oregano was subjected to drip irrigation, the water deficit being assessed in the vegetative and pre-flowering stages, as well as whole cycle analysis, through water matric potentials in the soil. The matric potential $-60.8 \mathrm{kPa}$ adopted in the irrigation handling during the oregano cultivation interval, led to higher essential oil content and yield. The same potential applied during the oregano pre-flowering stage resulted in the best mean of oregano fresh biomass production. The best dry biomass production was reached by using the matric potential $-91.2 \mathrm{kPa}$ in the oregano pre-flowering irrigation management. Water restriction in the soil throughout the entire phenological cycle favoured essential oil production, whereas the water restriction during the pre-flowering stage enabled high oregano dry biomass production. The highest values for biomass and essential oil productions were reached for the oregano plants cultivated during spring/summer.
\end{abstract}

Keywords: dielectric properties; drip; greenhouse production; soil water content; spice

\section{Introduction}

Oregano (Origanum vulgare L.) is an agronomic species of great productive potential worldwide due to its extensive eco-physiological amplitude (Landi and Guidi, 2015). This allogamous herbaceous angiosperm plant, besides its use as spice, has medicinal potential and presents $\mathrm{C} 3$ physiological mechanism. It belongs to family Lamiaceae and originally comes from the North-eastern coast of the Mediterranean Sea (Lukas et al., 2010; Dardioti et al., 2012; Bafana 2013; Raut and Karuppayil 2014; Do et al., 2015). This spice is mostly 
used in pasta recipes and is one of the main medicinal and spice plants grown in Germany and Argentina (Torres et al., 2012; Honermeier et al., 2013; Paraskevakis 2015). Oregano products have high added value, one kilogram of this spice costs $€ 180.00$, on average and one kilogram of its essential oil may cost $€ 6,000$ (Do et al., 2015).

Studies carried out since the early twenty-first century have shown that the oregano essential oil inhibits the growth of pathogenic microorganisms, such as Salmonella choleraesius, in the food (Souza et al., 2006), as well as of Aspergillus flavus, which contaminates table grapes at the postharvest stage (Sivakumar and Bautista-Bãnos, 2014). This oil also has nematicide effect against Meloidogyne incognita (Trivellini et al., 2016). On the other hand, the oregano extract has medicinal properties against breast and colon cancer cells (Savini et al., 2009; Guvenalp et al., 2010), as well as against the yellow fever causative agent (Meneses et al., 2009) and against diabetes mellitus (Lemhadri et al., 2004).

The main goal of irrigation in agriculture is to supply plants, mainly seasoning plants grown in protected environments, with water at the proper time and amount. Water content changes in the soil may affect the plant's vegetative growth and lead to decreased biomass and essential oil quality and productivity (Guidi and Landi, 2014). Innovative irrigation management techniques are acknowledged nowadays due to the current perspective about efficient water use. One of these techniques concerns the irrigation management based on using the water deficit, which is possible by monitoring the water content in the soil through the matric potential and the soil water dielectric constant (Whalley et al., 2013; Létourneau et al., 2015).

The water deficit in the soil may be directly monitored through time domain reflectometry (TDR). The soil water dielectric constant is measured through the TDR calibration equation, which is applied to determine the volumetric soil moisture. This constant allows finding the soil water matric potential expressed by the soil water retention curve, which is calculated through the van Genuchten equation. Such procedure quantifies the irrigation to be applied in agricultural cultivation (Bahreininejad et al., 2013; Batista et al., 2013; Gomes et al., 2014).

Oregano plants have been the core topic of scientific investigations in the plant nutrition and drug synthesis field, since they grow in places lacking rainfall in summer; however, studies about the response of these plants to water deficit are not found in the literature. There is evidence that this seasoning species is able to support high water deficit levels in the soil. Therefore, the aim of the present study was to investigate the biomass production and essential oil biosynthesis responses under different water availability conditions in order to set the optimum water matric potential for oregano irrigation handling and establish the best season to produce essential oil and biomass of Origanum vulgare L.

\section{Materials and Methods}

\section{Experimental tests}

Two experiments were conducted in a protected environment of the Biosystems Engineering Department, at University of São Paulo (USP), Piracicaba County - São Paulo State, Brazil (22 $42^{\prime}$ S; $47^{\circ} 37^{\prime}$ W; altitude $550 \mathrm{~m})$. An arch-type greenhouse $(7.0 \mathrm{~m}$ wide, $22.5 \mathrm{~m}$ long and $3.0 \mathrm{~m}$ high) was used to implement the experiments. The first experiment was conducted between November $6^{\text {th }}, 2014$ and February $9^{\text {th }}, 2015$ (spring/summer). The second one was conducted between May 14 $4^{\text {th }}, 2015$ and August $17^{\text {th }}, 2015$ (autumn/winter).

The experiments followed the factorial randomized block design, with additional treatment (Healy, 1956). The factorial design consisted of four soil water matric potentials $(-60.8 ;-91.2 ;-121.2$ and $-152.0 \mathrm{kPa})$ adopted as soil irrigation management reference, which was applied in three different cultivation stages (whole cycle, vegetative stage and pre-flowering stage) (Matraka et al., 2010; Chauhan et al., 2013). The 
control treatment $(-19.7 \mathrm{kPa})$ was run throughout the oregano cultivation cycle, because its matric potential corresponded to the field capacity of the soil. The cultivation stages were defined as follows: whole cycle, which lasted from seedling transplantation to harvesting; vegetative stage, from seedling transplantation to the emergence of the first floral branch; pre-flowering stage, from floral branch emission to full floweropening (Davidenco et al., 2015).

Each treatment consisted of six repetitions, thus totaling 78 plots. Each plot corresponded to a 12-liter conical trunk-shaped pot containing one Origanum vulgare L. plant.

\section{Irrigation planning and management}

The water content in the soil was monitored through time domain reflectometry (Field Scout TDR 100). The equipment was calibrated by using the soil water dielectric constant (ka) before conducting the experiments under volumetric soil moisture conditions (Gomes et al., 2014). A soil sample was removed from the pot after each ka reading; an auger screw was used to determine the volumetric water content (Uhland, 1951). Non-deformed soil samples were collected from the pots subjected to TDR calibrations, which were conducted to generate the soil water retention curves (van Genutchen, 1980). The soil used in the experiments is classified as Latossolo Vermelho eutrofico tipico, in Brazil, (Embrapa, 2013) and as Typic Hapludox, in the United States (Soil Survey Staff, 1999).

A trickle irrigation system, whose drip emitters presented $2 \mathrm{~L} \mathrm{~h}^{-1}$ nominal flow rate (Naan Daan Jain Taper lock outlet $2.0 \mathrm{kgf} \mathrm{cm}^{-2}$ ) was adopted. The lateral and derivation lines were composed of polyethylene hose (13 mm nominal diameter - ND) - the main line of the system was installed using a 19-mm-ND polyvinyl chloride pipe (PVC). Ball valves were installed at the beginning of each derivation line to independently irrigate the treatments. The volumetric moisture spatial variability in the plots was minimized by using a pair of microtubes ( $4 \mathrm{~mm}$ internal diameter) coupled to each dripper. The plant grew between a pair of water emission points (Batista et al., 2013).

Irrigation was performed when the water content in the plots reached volumetric moisture corresponding to the matric potential of the treatment. It was done in order to enable the soil to achieve its field capacity, at the end of the irrigation event. Daily readings of the water dielectric constant in the soil (ka) were carried out during the experiments (Xu et al., 2007). Handheld probes were vertically inserted into the pots down to the last soil layer $(0.20 \mathrm{~m}$ depth).

All treatments were kept in soil water matric potential $-19.7 \mathrm{kPa}$ (field capacity) throughout the first two cultivation weeks in order to assure seedling acclimation and to standardize the volumetric soil moisture conditions. The irrigation management under water deficit conditions was performed fifteen days after seedling transplantation (Bekhradi et al., 2015).

The herein found ka values allowed obtaining the current volumetric soil moisture value (Equation 1). The soil water matric potential was calculated through Equation 2 (van Genutchen 1980) by using the volumetric soil moisture value, which was determined through the adoption of the independent variable TDR. The irrigation time was calculated through Equation 3 (Bahreininejad et al., 2013).

Acurrent $=0.0798208 \cdot k a+2.255078$

$$
\begin{aligned}
& \text { Acurrent }=0.0780+\frac{0.3520}{\left[1+\left(\psi_{m}\right)^{1.5600}\right]^{0.3590}} \\
& T_{i}=\frac{\left(\theta_{f c}-\theta_{\text {current }}\right) \cdot D_{\text {ef }} A}{E_{a} \cdot Q_{\text {actual }}}
\end{aligned}
$$

wherein: 
$\theta_{\text {current }}=$ current volumetric soil moisture, $\mathrm{cm}^{3} \mathrm{~cm}^{-3}$;

$\mathrm{ka}=$ soil water dielectric constant, dimensionless;

$\Psi \mathrm{m}=$ current soil water matric potential $|\mathrm{kPa}|$;

$\theta_{\mathrm{fC}}=$ volumetric soil moisture at field capacity, $\mathrm{cm}^{3} \mathrm{~cm}^{-3}$;

$\mathrm{D}_{\text {ef }}=$ effective root depth, $\mathrm{mm}$;

$\mathrm{T}_{\mathrm{i}}=$ Irrigation time, hours;

$A=$ pot surface area, $\mathrm{m}^{2}$;

$\mathrm{E}_{\mathrm{a}}=$ irrigation system application efficiency, \%; and

$\mathrm{Q}_{\text {actual }}=$ actual emitter flow set through the uniformity and flow tests, $\mathrm{L} \mathrm{h}^{-1}$.

\section{Oregano plant cultivation}

The oregano seedlings were transplanted at the 33rd-day of life. The meteorological variables (air temperature and relative humidity) were measured every one hour and monitored throughout the whole experiment. These variables were recorded in an automatic thermo-hygrograph using a data logger (HT 4000 Hiseg model) located in the center of the greenhouse, according to the methodology of Batista et al. (2013).

The harvesting was performed 96 days after seedling transplantation. Leaves and inflorescences were individually subjected to manual collection, packaged in kraft paper bags and dried in forced ventilation oven, at $40{ }^{\circ} \mathrm{C}$, for 96 hours (Economakis, 1993). The biomass was weighed in analytical scale, before and after drying, to measure shoot fresh and dry weight, respectively.

The oregano essential oil was extracted through steam hydro-distillation (Clevenger, 1928; Voltolina, 2017). Dry shoot biomass was added to $1,500 \mathrm{ml}$ of distilled water and the mixture was placed in a 2 -liter volumetric flask. Subsequently, the mixture was heated in thermal blanket at $100{ }^{\circ} \mathrm{C}$, for 90 minutes (Asensio et al., 2015). The obtained essential oil mass was measured after the oil was extracted; the remaining water was decanted at $4{ }^{\circ} \mathrm{C}$ for 48 hours (Busatta et al., 2007). The essential oil content was set by the extracted oil mass/shoot dry mass ratio (El Gendy et al., 2015; Voltolina, 2017).

\section{Statistical analysis}

Analysis of variance (ANOVA) were performed to assess biomass and essential oil data, at 5\% significance level. In case of significant effects, LSD test was performed to compare the different mean soil water matric potentials and the three phenological stages assessed in the R software (Breusch and Pagan, 1979; Box et al., 2005; R Development Core Team, 2013).

\section{Results}

\section{Biomass production}

The statistical interaction between the soil water matric potential and the oregano phenological stages $(\mathrm{p}<0.01)$ was checked and the best mean biomass production was not found based on the lower or higher matric potentials assessed in the current study. Plants subjected to hydric limitation in all phenological stages of the first experiment, recorded the highest mean fresh biomass $(\mathrm{p}<0.05)$ at matric potential higher than $100 \mathrm{kPa}$, with emphasis to the means recorded in the pre-flowering stage (Table 1). The second experiment recorded the best means in plants subjected to hydric limitation during the pre-flowering (matric potential higher than $-100 \mathrm{kPa}$ ) and vegetative stages (matric potential higher than $-65 \mathrm{kPa})(\mathrm{p}<0.01)($ Table 1$)$.

The highest mean dry biomasses were recorded by plants cultivated under water deficit in spring/summer (Experiment 1) and treated with matric potential $-91.2 \mathrm{kPa}$ in the pre-flowering stage $(\mathrm{p}<$ 0.01) (Table 2). The dry biomass from the fall/winter cultivation (Experiment 2) showed significant difference in the oregano phenological stage $(\mathrm{p}<0.05)$; the means of plants facing water restriction in the pre-flowering stage (Table 2) were $22 \%$ higher than those of other plants. 
The control plants grown in spring/summer (Experiment 1) showed statistical fresh and dry weight mean and standard error $70.32 \pm 4.59$ and $16.17 \pm 1.13$ grams, respectively. These means were statistically different $(p<0.05)$ from the mean values shown by plants grown under water limitation. The experiment carried out in the autumn/winter season did not show mean dry and fresh mass differences between the control and water deficit treatments $(\mathrm{p}>0.05)$. The control plants showed mean fresh weight $46.11 \pm 5.99$ grams and mean dry matter $14.99 \pm 2.09$ grams.

The experiments conducted with plants cultivated in soil facing restricted water availability in the phenological pre-flowering stage, at matric potential $-91.2 \mathrm{kPa}$, recorded the best mean dry biomass. The experiments conducted with plants cultivated in soil facing restricted water availability in the phenological pre-flowering stage and matric potential $-60,8 \mathrm{kPa}$ have presented the best mean fresh biomass. The season presenting the bests means was spring/summer $(\mathrm{p}<0.05$ - fresh biomass; $\mathrm{p}<0.05$ - dry biomass) (Tables 3 and 4).

Table 1. Origanum vulgare L. fresh biomass production within the experiments

\begin{tabular}{|c|c|c|c|c|c|}
\hline \multirow{2}{*}{ Phenological atage } & \multicolumn{4}{|c|}{ Matric potential $(-\mathrm{kPa})$} & \multirow{2}{*}{ Mean } \\
\hline & 60.8 & 91.2 & 121.6 & 152.0 & \\
\hline \multicolumn{6}{|c|}{ Experiment 1 (spring/summer $-11 / 6^{\text {th }} / 2014$ to $\left.02 / 9^{\text {th }} / 2015\right)$} \\
\hline Pre-flowering & $67.51 \pm 4.99 \mathrm{~A} \mathrm{a}$ & $64.62 \pm 4.04 \mathrm{~A} \mathrm{a}$ & $56.88 \pm 3.85 \mathrm{~B} \mathrm{a}$ & $55.41 \pm 1.84 \mathrm{~B} \mathrm{a}$ & $60.42 \pm 3.52$ \\
\hline Whole cycle & $61.05 \pm 1.14 \mathrm{~A} \mathrm{a}$ & $62.14 \pm 1.09 \mathrm{~A} \mathrm{a}$ & $6.79 \pm 0.10 \mathrm{~B} \mathrm{~b}$ & $6.44 \pm 0.28 \mathrm{~B} \mathrm{~b}$ & $34.13 \pm 0.40$ \\
\hline Vegetative stage & $62.18 \pm 2.35 \mathrm{~A} \mathrm{a}$ & $61.88 \pm 2.92 \mathrm{~A} \mathrm{a}$ & $6.88 \pm 0.33 \mathrm{~B} \mathrm{~b}$ & $6.26 \pm 0.30 \mathrm{~B} \mathrm{~b}$ & $34.96 \pm 2.32$ \\
\hline Mean & $63.58 \pm 2.83$ & $62.88 \pm 2.76$ & $23.52 \pm 1.51$ & $22.70 \pm 0.87$ & $43.17 \pm 1.56$ \\
\hline \multicolumn{6}{|c|}{ Experiment 2 (autumn/winter $-05 / 14^{\text {th }} / 2015$ to $\left.08 / 17^{\text {th }} / 2015\right)$} \\
\hline Pre-flowering & $46.34 \pm 4.57 \mathrm{~A} \mathrm{a}$ & $44.03 \pm 7.54 \mathrm{~A} \mathrm{a}$ & $35.93 \pm 3.84 \mathrm{~B} \mathrm{a}$ & $34.00 \pm 3.82 \mathrm{~B} \mathrm{a}$ & $40.08 \pm 4.17$ \\
\hline Whole cycle & $34.24 \pm 4.15 \mathrm{~A} \mathrm{~b}$ & $26.02 \pm 4.99 \mathrm{~B} \mathrm{c}$ & $13.58 \pm 1.61 \mathrm{Cb}$ & $11.07 \pm 1.15 \mathrm{C} \mathrm{b}$ & $20.80 \pm 2.75$ \\
\hline Vegetative stage & $44.02 \pm 1.05 \mathrm{~A} \mathrm{a}$ & $31.19 \pm 4.00 \mathrm{~B} \mathrm{~b}$ & $15.42 \pm 5.33 \mathrm{Cb}$ & $14.21 \pm 1.41 \mathrm{C} \mathrm{b}$ & $25.65 \pm 3.05$ \\
\hline Mean & $41.53 \pm 3.26$ & $33.75 \pm 5.51$ & $21.64 \pm 4.01$ & $19.79 \pm 3.71$ & $28.84 \pm 3.51$ \\
\hline
\end{tabular}

Note: Means (value \pm standard error) followed by lowercase letters in the column (Phenological Stage) or capital letters on the line (Matric Potential) significantly differ from each other in the LSD Test $(\mathrm{p}<0.05)$

Table 2. Origanum vulgare L. dry biomass production within the experiments

\begin{tabular}{|c|c|c|c|c|c|}
\hline \multirow{2}{*}{ Phenological stage } & \multicolumn{4}{|c|}{ Matric potential $(-\mathrm{kPa})$} & \multirow{2}{*}{ Mean } \\
\hline & 60.8 & 91.2 & 121.6 & 152.0 & \\
\hline \multicolumn{6}{|c|}{ Experiment 1 (spring/summer $-11 / 6^{\text {th }} / 2014$ to $\left.02 / 9^{\text {th }} / 2015\right)$} \\
\hline Pre-flowering & $15.88 \pm 1.39 \mathrm{AB} a$ & $21.20 \pm 0.83 \mathrm{~A} \mathrm{a}$ & $14.97 \pm 1.34 \mathrm{AB}$ a & $13.43 \pm 0.34 \mathrm{~B} \mathrm{a}$ & $16.37 \pm 0.58$ \\
\hline Whole cycle & $15.02 \pm 0.36 \mathrm{~B} \mathrm{a}$ & $18.60 \pm 0.64 \mathrm{~A} \mathrm{~b}$ & $1.69 \pm 0.05 \mathrm{C} \mathrm{b}$ & $1.55 \pm 0.11 \mathrm{C} \mathrm{b}$ & $9.22 \pm 1.09$ \\
\hline Vegetative stage & $14.72 \pm 1.01 \mathrm{~B} \mathrm{a}$ & $15.37 \pm 0.37 \mathrm{Ac}$ & $1.81 \pm 0.13 \mathrm{C} \mathrm{b}$ & $1.79 \pm 0.06 \mathrm{C} \mathrm{b}$ & $8.42 \pm 0.88$ \\
\hline Mean & $15.21 \pm 0.57$ & $18.39 \pm 1.33$ & $5.82 \pm 1.41$ & $5.59 \pm 1.55$ & $11.33 \pm 0.55$ \\
\hline \multicolumn{6}{|c|}{ Experiment 2 (autumn/winter - $05 / 14^{\text {th }} / 2015$ to $\left.08 / 17^{\text {th }} / 2015\right)$} \\
\hline Pre-flowering & $14.80 \pm 0.89$ & $15.66 \pm 1.97$ & $13.22 \pm 1.11$ & $16.36 \pm 2.53$ & $15.01 \pm 0.90 \mathrm{a}$ \\
\hline Whole cycle & $12.14 \pm 1.52$ & $11.91 \pm 1.19$ & $11.87 \pm 5.45$ & $10.01 \pm 1.46$ & $11.48 \pm 1.19 \mathrm{~b}$ \\
\hline Vegetative stage & $12.59 \pm 2.00$ & $12.02 \pm 0.94$ & $12.13 \pm 1.21$ & $12.97 \pm 2.50$ & $12.43 \pm 1.08 \mathrm{~b}$ \\
\hline Mean & $13.18 \pm 0.88 \mathrm{~A}$ & $13.19 \pm 0.89 \mathrm{~A}$ & $12.40 \pm 1.17 \mathrm{~A}$ & $13.11 \pm 1.06 \mathrm{~A}$ & $12.95 \pm 0.69$ \\
\hline
\end{tabular}

Note: Means (value \pm standard error) followed by lowercase letters in the column (Phenological Stage) or capital letters on the line (Matric Potential) significantly differ from each other in the LSD test $(\mathrm{p}<0.05)$

Table 3. Season effect on each Origanum vulgare L. dry biomass treatment

$$
\text { Dry biomass }(\mathrm{g})
$$


Thaner Dos Santos H et al. (2020). Not Sci Biol 12(1):42-56.

\begin{tabular}{|c|c|c|c|c|}
\hline \multirow[b]{2}{*}{ Phenological stage } & \multirow{2}{*}{$\begin{array}{c}\text { Matric } \\
\text { potential } \\
(-\mathrm{kPa})\end{array}$} & Spring/summer & Autumn/winter & \multirow[b]{2}{*}{ Mean } \\
\hline & & $\begin{array}{c}11 / 6^{\text {th }} / 2014 \text { to } \\
02 / 9^{\text {th }} / 2015\end{array}$ & $\begin{array}{c}05 / 14^{\text {th }} / 2015 \text { to } \\
08 / 17^{\text {th }} / 2015\end{array}$ & \\
\hline \multirow{4}{*}{ Whole cycle } & 60.8 & $15.02 \pm 0.36 \mathrm{~A} \mathrm{~b}$ & $12,14 \pm 0,89 \mathrm{~B} \mathrm{~b}$ & $13.58 \pm 0.58$ \\
\hline & 91.2 & $18.60 \pm 0.64 \mathrm{~A} \mathrm{~b}$ & $11.91 \pm 1.19 \mathrm{~B} \mathrm{~b} \mathrm{c}$ & $15.26 \pm 0.85$ \\
\hline & 121.2 & $1.69 \pm 0.05 \mathrm{~B} \mathrm{~d}$ & $11.87 \pm 5.45 \mathrm{~A} \mathrm{bc}$ & $6.78 \pm 2.56$ \\
\hline & 152 & $1.55 \pm 0.11 \mathrm{~B} \mathrm{~d}$ & $10.01 \pm 1.46 \mathrm{Ac}$ & $5.78 \pm 0.73$ \\
\hline \multirow{4}{*}{ Vegetative stage } & 60.8 & $14.72 \pm 1.01 \mathrm{~A} \mathrm{~b}$ & $12.59 \pm 2.00 \mathrm{~A} \mathrm{a} \mathrm{b}$ & $13.66 \pm 1.40$ \\
\hline & 91.2 & $15.37 \pm 0.37 \mathrm{~A} \mathrm{~b}$ & $12.02 \pm 0.94 \mathrm{~B} \mathrm{~b}$ & $13.70 \pm 0.61$ \\
\hline & 121.2 & $1.81 \pm 0.13 \mathrm{~B} \mathrm{~d}$ & $12.13 \pm 1.21 \mathrm{Ab}$ & $6.97 \pm 0.62$ \\
\hline & 152 & $1.79 \pm 0.06 \mathrm{~B} \mathrm{~d}$ & $12.97 \pm 2.50 \mathrm{~A} \mathrm{a} \mathrm{b}$ & $7.38 \pm 1.19$ \\
\hline \multirow{4}{*}{ Pre-flowering } & 60.8 & $15.88 \pm 1.39 \mathrm{~A} \mathrm{~b}$ & $14.80 \pm 0.89 \mathrm{~A} \mathrm{a}$ & $15.34 \pm 1.06$ \\
\hline & 91.2 & $21.20 \pm 0.83 \mathrm{~A} \mathrm{a}$ & $15.66 \pm 1.97 \mathrm{~B} \mathrm{a}$ & $18.43 \pm 1.30$ \\
\hline & 121.2 & $14.97 \pm 1.34 \mathrm{~A} \mathrm{~b}$ & $13.22 \pm 1.11 \mathrm{~B} \mathrm{ab}$ & $14.10 \pm 1.14$ \\
\hline & 152 & $13.43 \pm 0.34 \mathrm{~B} \mathrm{~b}$ & $16.36 \pm 2.53 \mathrm{~A} \mathrm{a}$ & $14.90 \pm 1.33$ \\
\hline \multicolumn{2}{|c|}{ Control } & $16.17 \pm 1,13 \mathrm{~A} \mathrm{a} \mathrm{b}$ & $14.99 \pm 2.09 \mathrm{~A} \mathrm{a}$ & $15.58 \pm 1.61$ \\
\hline \multicolumn{2}{|c|}{ Mean } & $11.71 \pm 0.60$ & $13.13 \pm 1.86$ & $12.42 \pm 1.23$ \\
\hline
\end{tabular}

Note: Means (value \pm standard error) followed by capital letters on the line (seasonal season) and by lowercase letters in the column (treatments) significantly differ from each other in the LSD test $(\mathrm{p}<0.05)$

Table 4. Season effect on each Origanum vulgare L. fresh biomass treatment

\begin{tabular}{|c|c|c|c|c|}
\hline \multicolumn{5}{|c|}{ Fresh biomass (g) } \\
\hline \multirow[b]{2}{*}{ Phenological stage } & \multirow{2}{*}{$\begin{array}{c}\text { Matric } \\
\text { Potential } \\
(-\mathrm{kPa})\end{array}$} & Spring/summer & Autumn/winter & \multirow[b]{2}{*}{ Mean } \\
\hline & & $\begin{array}{l}11 / 06^{\text {th }} / 2014 \text { to } \\
02 / 09^{\text {th }} / 2015\end{array}$ & $\begin{array}{c}05 / 14^{\text {th }} / 2015 \text { to } \\
08 / 17^{\text {th }} / 2015\end{array}$ & \\
\hline \multirow{4}{*}{ Whole cycle } & 60.8 & $61.05 \pm 1.14 \mathrm{Abc}$ & $34.24 \pm 4.15 \mathrm{~B} \mathrm{a}$ & $47.65 \pm 2.63$ \\
\hline & 91.2 & $62.14 \pm 1.09 \mathrm{~A} \mathrm{bc}$ & $26.02 \pm 4.99 \mathrm{~B} \mathrm{~d}$ & $44.08 \pm 3.59$ \\
\hline & 121.2 & $6.79 \pm 0.10 \mathrm{~B} \mathrm{~d}$ & $11.87 \pm 5.45 \mathrm{Af}$ & $9.33 \pm 2.28$ \\
\hline & 152 & $6.44 \pm 0.28 \mathrm{~B} \mathrm{~d}$ & $11.07 \pm 1.15 \mathrm{~A} \mathrm{f}$ & $8.76 \pm 1.70$ \\
\hline \multirow{4}{*}{ Vegetative stage } & 60.8 & $62.18 \pm 2.35 \mathrm{~A} \mathrm{~b} \mathrm{c}$ & $44.02 \pm 1.05 \mathrm{~B} \mathrm{a}$ & $53.1 \pm 3.79$ \\
\hline & 91.2 & $61.88 \pm 2.92 \mathrm{~A} \mathrm{bc}$ & $31.19 \pm 4.00 \mathrm{~B} \mathrm{c}$ & $46.54 \pm 3.46$ \\
\hline & 121.2 & $6.88 \pm 0.33 \mathrm{~B} \mathrm{~d}$ & $15.42 \pm 5.33 \mathrm{~A} \mathrm{e}$ & $11.15 \pm 3.38$ \\
\hline & 152 & $6.26 \pm 0.30 \mathrm{~B} \mathrm{~d}$ & $11.97 \pm 2.50 \mathrm{Af}$ & $9.12 \pm 2.73$ \\
\hline \multirow{4}{*}{ Pre-flowering } & 60.8 & $67.51 \pm 4.99 \mathrm{~A} \mathrm{a} \mathrm{b}$ & $46.34 \pm 4.57 \mathrm{~B} \mathrm{a}$ & $56.93 \pm 2.39$ \\
\hline & 91.2 & $64.62 \pm 4.04 \mathrm{~A} \mathrm{a} \mathrm{b}$ & $44.03 \pm 7.54 \mathrm{~B} \mathrm{a}$ & $54.33 \pm 5.01$ \\
\hline & 121.2 & $56.88 \pm 3.85 \mathrm{Ac}$ & $35.93 \pm 3.84 \mathrm{~B} \mathrm{a}$ & $46.41 \pm 2.98$ \\
\hline & 152 & $55.41 \pm 1.84 \mathrm{Ac}$ & $34.00 \pm 3.82 \mathrm{~B} \mathrm{~b}$ & $44.71 \pm 2.17$ \\
\hline \multicolumn{2}{|c|}{ Control } & $70.32 \pm 4.59 \mathrm{~A} \mathrm{a}$ & $46.11 \pm 5.99 \mathrm{~B} \mathrm{a}$ & $58.22 \pm 4.29$ \\
\hline \multicolumn{2}{|c|}{ Mean } & $45.26 \pm 2.04$ & $30.17 \pm 4.78$ & $37.71 \pm 3.19$ \\
\hline
\end{tabular}

Note: Means (value \pm standard error) followed by capital letters on the line (seasonal season) and by lowercase letters in the column (treatments) significantly differ from each other in the LSD test $(\mathrm{p}<0.05)$

\section{Essential oil biosynthesis}

The best mean essential oil content in both experiments was recorded by plants whose soil water matric potential was $-60.8 \mathrm{kPa}$, during the whole cultivation cycle (Tables 5 and 6). Plants in Experiment 2 (fall/winter), which were cultivated under water restriction in the pre-flowering stage and treated with matric potential higher than $-125 \mathrm{kPa}$, presented mean essential oil content statistically equal to plants cultivated under soil water limitation throughout the whole phenological cycle, at matric potential $-60.8 \mathrm{kPa}$ (Table 5). 
48

Similar result was recorded for essential oil yield; plants subjected to soil water limitation during the preflowering stage, at matric potential $-91.2 \mathrm{kPa}$, showed high mean essential oil yield (Table 6).

Plants cultivated in soil with water matric potential between $-90 \mathrm{kPa}$ and $-125 \mathrm{kPa}$, during the preflowering stage in both experiments, presented essential oil means higher than those recorded in plants subjected to water restriction in the vegetative stage (Tables 5 and 6). Plants cultivated under water restriction in the vegetative stage or throughout the whole cultivation cycle, whose soil water matric potential for irrigation was lower than $-100 \mathrm{kPa}$, did not produce essential oil.

The highest essential oil yield and content indices were reached in Experiment 1, which was performed in the spring/summer season ( $\mathrm{p}<0.01$ - content; $\mathrm{p}<0.01$ - yield) (Tables 7 and 8).

Table 5. Origanum vulgare L. essential oil content within the experiments

\begin{tabular}{|c|c|c|c|c|}
\hline \multirow{3}{*}{$\begin{array}{l}\text { Matric potential } \\
\qquad(-\mathrm{kPa})\end{array}$} & \multirow{3}{*}{ Phenological stage } & \multicolumn{2}{|c|}{ Essential oil content (\%) } & \multirow{3}{*}{ Mean } \\
\hline & & Spring/summer & Autumn/winter & \\
\hline & & $\begin{array}{c}11 / 06^{\text {th }} / 2014 \text { to } \\
02 / 09^{\text {th }} / 2015\end{array}$ & $\begin{array}{c}05 / 14^{\text {th }} / 2015 \text { to } \\
08 / 17^{\text {th }} / 2015\end{array}$ & \\
\hline 60.8 & Whole cycle & $3.080 \pm 0.074 \mathrm{a}$ & $0.243 \pm 0.180 \mathrm{a}$ & $1,661 \pm 0,641$ \\
\hline 60.8 & Vegetative stage & $0.420 \pm 0.021 \mathrm{~d}$ & $0.120 \pm 0.014 \mathrm{c}$ & $0,270 \pm 0,103$ \\
\hline 60.8 & Pre-flowering & $0.536 \pm 0.036 c$ & $0.146 \pm 0.006 \mathrm{bc}$ & $0,341 \pm 0,093$ \\
\hline 91.2 & Vegetative stage & $0.130 \pm 0.015 \mathrm{e}$ & $0.173 \pm 0.019 \mathrm{bc}$ & $0,152 \pm 0,014$ \\
\hline 91.2 & Pre-flowering & $0.623 \pm 0.050 \mathrm{c}$ & $0.241 \pm 0.065 \mathrm{a}$ & $0,431 \pm 0,093$ \\
\hline 121.2 & Pre-flowering & $0.603 \pm 0.030 c$ & $0.240 \pm 0.021 \mathrm{a}$ & $0,422 \pm 0,071$ \\
\hline 152.0 & Pre-flowering & $0.580 \pm 0.049 c$ & $0.150 \pm 0.068 \mathrm{bc}$ & $0,365 \pm 0,081$ \\
\hline \multicolumn{2}{|c|}{ Control } & $0.843 \pm 0.090 \mathrm{~b}$ & $0.190 \pm 0.059 \mathrm{~b}$ & $0,516 \pm 0,154$ \\
\hline \multicolumn{2}{|c|}{ Mean } & $0.852 \pm 0.180$ & $0.189 \pm 0.024$ & $0.520 \pm 0.102$ \\
\hline \multicolumn{2}{|c|}{ P-value } & $<0.002$ & $<0.009$ & - \\
\hline
\end{tabular}

Note: Mean (value \pm standard error) followed by lowercase letters in the column significantly differ from each other in the LSD test $(\mathrm{p}<0.05)$

Table 6. Origanum vulgare L. essential oil yield within the experiments

\begin{tabular}{|c|c|c|c|c|}
\hline \multirow{3}{*}{$\begin{array}{l}\text { Matric potential } \\
\qquad(-\mathrm{kPa})\end{array}$} & \multirow{3}{*}{ Phenological stage } & \multicolumn{2}{|c|}{ essential oil yield (mg plant $\left.{ }^{-1}\right)$} & \multirow{3}{*}{ Mean } \\
\hline & & Spring/summer & Autumn/winter & \\
\hline & & $\begin{array}{c}11 / 06^{\text {th }} / 2014 \text { to } \\
02 / 09^{\text {th }} / 2015\end{array}$ & $\begin{array}{c}05 / 14^{\text {th }} / 2015 \text { to } \\
08 / 17^{\text {th }} / 2015\end{array}$ & \\
\hline 60.8 & Whole cycle & $26.25 \pm 5.94 \mathrm{a}$ & $3.12 \pm 1.37 \mathrm{a}$ & $14.71 \pm 3.55$ \\
\hline 60.8 & Vegetative stage & $7.88 \pm 0.48 \mathrm{~cd}$ & $1.48 \pm 0.28 \mathrm{de}$ & $4.71 \pm 1.00$ \\
\hline 60.8 & Pre-flowering & $6.68 \pm 1.18 \mathrm{~d}$ & $2.10 \pm 0.22 \mathrm{~cd}$ & $4.26 \pm 0.81$ \\
\hline 91.2 & Vegetative stage & $2.74 \pm 0.42 \mathrm{e}$ & $2.00 \pm 0.26 \mathrm{cde}$ & $2.39 \pm 0.14$ \\
\hline 91.2 & Pre-flowering & $9.41 \pm 1.46 \mathrm{c}$ & $2.76 \pm 0.42 \mathrm{ab}$ & $6.10 \pm 1.03$ \\
\hline 121.2 & Pre-flowering & $8.07 \pm 2.40 \mathrm{~cd}$ & $2.31 \pm 0.13 \mathrm{bcd}$ & $5.19 \pm 1.11$ \\
\hline 152.0 & Pre-flowering & $8.04 \pm 1.34 \mathrm{~cd}$ & $2.10 \pm 0.35 \mathrm{~cd}$ & $5.07 \pm 0.82$ \\
\hline \multicolumn{2}{|c|}{ Control } & $14.04 \pm 0.86 b$ & $2.69 \pm 0.38 b$ & $8.39 \pm 1.76$ \\
\hline \multicolumn{2}{|c|}{ Mean } & $10.38 \pm 1.84$ & $2.32 \pm 0.28$ & $6.35 \pm 1.07$ \\
\hline \multicolumn{2}{|c|}{ P-value } & $<0.009$ & $<0.0004$ & - \\
\hline
\end{tabular}

Note: Means (value \pm standard error) followed by lowercase letters in the column significantly differ from each other in the LSD test $(\mathrm{p}<0.05)$ 
Thaner Dos Santos H et al. (2020). Not Sci Biol 12(1):42-56.

Table 7. Season effects on the content of Origanum vulgare L. essential oil

\begin{tabular}{|c|c|c|c|c|}
\hline \multirow{3}{*}{$\begin{array}{l}\text { Matric potential } \\
\qquad(-\mathrm{kPa})\end{array}$} & \multirow{3}{*}{ Phenological stage } & \multicolumn{2}{|c|}{ Essential oil content (\%) } & \multirow{3}{*}{ Mean } \\
\hline & & Spring/summer & Autumn/winter & \\
\hline & & $\begin{array}{c}11 / 06^{\text {th }} / 2014 \text { to } \\
02 / 09^{\text {th }} / 2015\end{array}$ & $\begin{array}{c}05 / 14^{\text {th }} / 2015 \text { to } \\
08 / 17^{\text {th }} / 2015\end{array}$ & \\
\hline 60.8 & Whole cycle & $3.080 \pm 0.074 \mathrm{~A} \mathrm{a}$ & $0.243 \pm 0.180 \mathrm{~B} \mathrm{a}$ & $1,661 \pm 0,641$ \\
\hline 60.8 & Vegetative stage & $0.420 \pm 0.021 \mathrm{~A} \mathrm{~d}$ & $0.120 \pm 0.014 \mathrm{~B} \mathrm{a}$ & $0,270 \pm 0,103$ \\
\hline 60.8 & Pre-flowering & $0.536 \pm 0.036 \mathrm{Acd}$ & $0.146 \pm 0.006 \mathrm{~B} \mathrm{a}$ & $0,341 \pm 0,093$ \\
\hline 91.2 & Vegetative stage & $0.130 \pm 0.015 \mathrm{~A} \mathrm{e}$ & $0.173 \pm 0.019 \mathrm{~A} \mathrm{a}$ & $0,152 \pm 0,014$ \\
\hline 91.2 & Pre-flowering & $0.623 \pm 0.050 \mathrm{Ac}$ & $0.241 \pm 0.065 \mathrm{~B} \mathrm{a}$ & $0,431 \pm 0,093$ \\
\hline 121.2 & Pre-flowering & $0.603 \pm 0.030 \mathrm{~A} \mathrm{c}$ & $0.240 \pm 0.021 \mathrm{~B} \mathrm{a}$ & $0,422 \pm 0,071$ \\
\hline 152.0 & Pre-flowering & $0.580 \pm 0.049 \mathrm{~A} \mathrm{c} \mathrm{d}$ & $0.150 \pm 0.068 \mathrm{~B} \mathrm{a}$ & $0,365 \pm 0,081$ \\
\hline \multicolumn{2}{|c|}{ Control } & $0.843 \pm 0.090 \mathrm{~A} \mathrm{~b}$ & $0.190 \pm 0.059 \mathrm{~B} \mathrm{a}$ & $0,516 \pm 0,154$ \\
\hline \multicolumn{2}{|c|}{ Mean } & $0.852 \pm 0.180$ & $0.189 \pm 0.024$ & $0.520 \pm 0.102$ \\
\hline
\end{tabular}

Note: Means (value \pm standard error) followed by capital letters on the line (seasonal period) and lowercase letters in the columns (treatments) significantly differed from each other in the LSD test $(\mathrm{p}<0.05)$

Table 8. Season effect on the yield of Origanum vulgare L. essential oil

\begin{tabular}{|c|c|c|c|c|}
\hline \multirow{3}{*}{$\begin{array}{l}\text { Matric potential } \\
\qquad(-\mathrm{kPa})\end{array}$} & \multirow{3}{*}{ Phenological stage } & \multicolumn{2}{|c|}{ Essential oil yield $\left(\mathrm{mg}\right.$ plant $\left.^{-1}\right)$} & \multirow{3}{*}{ Mean } \\
\hline & & Spring/summer & Autumn/winter & \\
\hline & & $\begin{array}{c}11 / 06^{\text {th }} / 2014 \text { to } \\
02 / 09^{\text {th }} / 2015\end{array}$ & $\begin{array}{c}05 / 14^{\text {th }} / 2015 \text { to } \\
08 / 17^{\text {th }} / 2015\end{array}$ & \\
\hline 60.8 & Whole cycle & $26.25 \pm 5.94 \mathrm{~A} \mathrm{a}$ & $3.12 \pm 1.37 \mathrm{~B} \mathrm{a}$ & $14.71 \pm 3.55$ \\
\hline 60.8 & Vegetative stage & $7.88 \pm 0.48 \mathrm{~A} \mathrm{~d} \mathrm{e}$ & $1.48 \pm 0.28 \mathrm{~B} \mathrm{e}$ & $4.71 \pm 1.00$ \\
\hline 60.8 & Pre-flowering & $6.68 \pm 1.18 \mathrm{~A} \mathrm{e}$ & $2.10 \pm 0.22 \mathrm{~B} \mathrm{c} \mathrm{d}$ & $4.26 \pm 0.81$ \\
\hline 91.2 & Vegetative stage & $2.74 \pm 0.42 \mathrm{Af}$ & $2.00 \pm 0.26 \mathrm{~B} \mathrm{~d}$ & $2.39 \pm 0.14$ \\
\hline 91.2 & Pre-flowering & $9.41 \pm 1.46 \mathrm{~A} \mathrm{c}$ & $2.76 \pm 0.42 \mathrm{~B} \mathrm{~d}$ & $6.10 \pm 1.03$ \\
\hline 121.2 & Pre-flowering & $8.07 \pm 2.40 \mathrm{~A} \mathrm{c} \mathrm{d}$ & $2.31 \pm 0.13 \mathrm{~B} \mathrm{c}$ & $5.19 \pm 1.11$ \\
\hline 152.0 & Pre-flowering & $8.04 \pm 1.34 \mathrm{Acd}$ & $2.10 \pm 0.35 \mathrm{~B} \mathrm{c} \mathrm{d}$ & $5.07 \pm 0.82$ \\
\hline \multicolumn{2}{|c|}{ Control } & $14.04 \pm 0.86 \mathrm{~A} \mathrm{~b}$ & $2.69 \pm 0.38 \mathrm{~B} \mathrm{~d}$ & $8.39 \pm 1.76$ \\
\hline \multicolumn{2}{|c|}{ Mean } & $10.38 \pm 1.84$ & $2.32 \pm 0.28$ & $6.35 \pm 1.07$ \\
\hline
\end{tabular}

Note: Means (value \pm standard error) followed by capital letters on the line (seasonal period) and by lowercase letters in the columns (treatments) significantly differed from each other in the LSD test $(\mathrm{p}<0.01)$

\section{Discussion}

\section{Biomass production}

The highest mean biomasses shown by plants subjected to soil water restriction did not result from the lowest or highest matric potentials assessed in the current study. The irrigation frequency based on different matric potentials enabled a wet and dry cycle that has positively influenced the nutrients, microbial fauna and root dynamics in the soil. Thus, the hormonal signaling from roots to shoots, as well as the organic matter mineralization and nutrient uptake, were positively potentiated (Dodd et al., 2015).

According to Du et al. (2015), the water deficit effects depend on the phenological stage, as well as on deficit duration and severity. A study conducted by Azizi et al. (2009) in Rauischholzhausen (Germany) investigated the soil water limitation effects on oregano plant production. They found that plants subjected to water deficit in the pre-flowering stage presented the highest mean biomasses. The study conducted by Baher et al. (2002) in Tehran (Iran) showed that Satureja hortensis L. subjected to water deficit in the preflowering stage recorded the best fresh and dry biomass production. Therefore, the irrigation handling 
50

focused on maintaining the water content in the soil at field capacity level, during the vegetative stage, makes roots and leaves save bioenergetical reserves to resist the water limitation (Osakabe et al., 2014).

One of the main plant physiological responses to soil water restriction lies on adjustments in biomass accumulation to end the phenological cycle. In other words, plants adjust their source-sink relation when they face environmental adversities; so, the energy expenditure can be minimized due to water absorption and the nutrient allocation in their structure can meet the suitable biochemical and physiological processes. In addition, plants may stiffen their cell wall to keep the turgor pressure and reduce the water losses caused by transpiration (Neumann, 1995; Shao et al., 2008).

The decreased emission of leaves and the reduced stomata opening frequency minimize water losses caused by evapotranspiration, and they are other examples of plant adaptation to soil water limitation. This process minimizes $\mathrm{CO}_{2}$ absorption; thus, mass accumulation reaches lower rates than those of plants that were not subjected to water-scarce environments (Chaves and Oliveira, 2004; Farooq et al., 2009; Seghatoleslami et al., 2015).

Khalil and El-Noemani (2015) assessed oregano plant growth based on different water depletion factors in Cairo, Egypt and found that plants whose irrigation depletion factor reference was 0.55, showed the highest mean fresh and dry biomasses. According to them, the combination of deficit irrigation and other cultural practices may promote biomass gain. Said-Al Ahl et al. (2009) assessed the response of oregano plants to different soil water depletion factors in Dokki (Egypt) and found that plants subjected to irrigation handling able to keep the water capacity of the soil in $80 \%$ showed the highest mean fresh and dry biomasses. They concluded that the irrigation applied to water deficit cases may improve the oregano fresh weight production rates.

The plants grown under irrigation deficit throughout the cultivation cycle and those grown under water deficit in the vegetative stage, at soil water matric potential higher than $-100 \mathrm{kPa}$, showed the highest mean fresh biomasses in the warmest season of the year - when the mean maximum and minimum temperatures reached $39.1{ }^{\circ} \mathrm{C} \pm 4.0^{\circ} \mathrm{C}$ and $20.2 \pm 2.3{ }^{\circ} \mathrm{C}$, respectively. The mean relative humidity was $58.7 \% \pm 5.6 \%$. Although the mean maximum temperature did not meet the temperature range required for oregano cultivation $\left(20-35^{\circ} \mathrm{C}\right.$ ) (Mijani et al., 2013), the culture showed high yield under spring and summer environmental conditions, because they activate the plant's secondary metabolic pathways, which are more efficient at this time of the year (Figueiredo et al., 2008).

According to Rioba et al. (2015), flavoring plants grown in different seasons show different responses. Ekren et al. (2012) conducted a study in Bornova,Turkey and found that the highest mean basil fresh and dry weights were recorded in the spring/summer season. According to them, the increased air temperature led to basil biomass gain.

Telci and Hisil (2008) found that coriander presents higher biomass production in spring, because the high temperatures stimulate leaf emission and enable greater light absorption. Farahani et al. (2009) assessed the performance of flavoring plants under water scarcity conditions in different seasons and found that the mass accumulation increased depending on the increased photoperiod and on the environmental temperature.

Kofidis et al. (2003) assessed the morphology and anatomy of oregano plants grown in different locations and seasons (in Greece) and found that plants grown in the spring/summer season recorded the highest mean biomass. They also found that these plants produced thick epidermis and mesophyll in the leaves when they met the evapotranspirometric demand of the season; therefore, water storage by leaves has helped cell turgor and, thereby, increased the leaf area. Thus, radiation absorption is expanded to positively boost biomass accumulation during the warmest seasons (Mortensen, 2014).

Finally, the response of oregano plants to water deficit is similar to the responses of other plants belonging to Lamiaceae family; thus, one can infer that oregano plants produce significant biomass when they 
are exposed to irrigation deficit, at soil water matric potential ranging from $-60 \mathrm{kPa}$ to $-95 \mathrm{kPa}$ in the preflowering stage, for irrigation management purposes.

\section{Essential oil production}

According to Penka (1978), the essential oil formation and accumulation in seasoning, hallucinogenic, medicinal and aromatic plants depends on two variables, one of them is the water content in the soil. The author pointed out that the lower the water content in the soil, the lower the essential oil biosynthesis by plants. However, irrigation tends to induce essential oil content and yield biosynthesis increase in plants, as long as it is combined with other factors such as the phenological water-stress period.

According to Hussein et al. (2014), seasoning plants subjected to water limitation show increased carbohydrate production due to source-sink relation adjustments in the plant, which leads to decreased essential oil production. Seasoning plants facing water limitation conditions overall adjust their source-sink relations so that the leaf mesophyll becomes the main sink, and the trichome, the supporting one. Consequently, the essential oil biosynthesis is reduced and biomass accumulation is favored by it (Burmeister and Guttenberg, 1960; Sangwan et al.2001).

Plants subjected to water limitation in the pre-flowering stage presented the highest mean biomass production rather than the highest essential oil production in the present study. Therefore, assumingly, plants respond to water limitation during the oil biosynthesis phenological stage by assuring biomass accumulation through water limitation as a way to keep the photosynthesis process running.

It is worth emphasizing that plants grown under matric potential lower than $-90 \mathrm{kPa}$ in the preflowering stage showed mean essential oil content and yield higher than plants grown under water limitation in the vegetative stage. According to Abdelmajeed et al. (2013) and Kofidis et al. (2003), the essential oil biosynthesis is not qualitatively and/or quantitatively constant; however, it helps plants to endure environmental limitations since the emission of glandular hair in the leaves during the pre-flowering stage helps reducing plant transpiration.

Hassan et al. (2013) assessed the deficit irrigation effects on protected rosemary cultivation in Taif ,Saudi Arabia and found that the plants, whose irrigation was managed at $60 \%$ field capacity, showed the highest mean essential oil content. They also discovered that water limitation leads to morphological adaptations developed to assure the plant's survival in the environment. The glandular hair increases in the leaves - which decreases transpiration and favors essential oil accumulation in the shoots - stands out among other adaptation factors.

Rioba et al. (2015) investigated the agronomic performance of sage (Salvia officinalis L.) by using different irrigation frequencies in Njoro, Kenya and found that plants irrigated every two weeks showed the highest mean essential oil contents. They also inferred that arid climate conditions influence essential oil synthesis by plants, because plants grown under higher irrigation frequency presented the highest essential oil content and yield.

Azizi et al. (2009) studied the water restriction effects on oregano plants grown in Rauischholzhausen ,Germany and found that the highest mean essential oil content and yield was shown by plants subjected to water limitation. Their results were similar to those found in the present study in plants subjected to matric potential higher than $-90 \mathrm{kPa}$ throughout the cultivation cycle.

The present results did not corroborate the study by Manukyan (2011), who assessed the essential oil yield of lemongrass plants in Munich. Germany and found that the highest mean essential oil content was recorded for treatments based on higher water limitation. However, he infers that the water retention capacity of the soil may or not attenuate the water deficit effects, and that such variable should be taken into consideration in future studies about water deficit effects on seasoning plants.

According to Abdelmajeed et al. (2013) and Figueiredo et al. (2008), variables such as essential oil content and yield depend on the cultivation environment the seasoning plant is subjected to. As previously 
mentioned, oregano plants achieve morphological adjustments in their organography based on the abiotic stress (Sangwan et al., 2011) and on the season (Kofidis et al., 2003).

Chang et al. (2005) reported that the essential oil yield by Lamiaceae plants is higher when the plant is grown under temperatures higher than $25^{\circ} \mathrm{C}$, which correspond to the warmest seasons. The plants in the present study showed essential oil yield under minimum and maximum temperatures between $13.1{ }^{\circ} \mathrm{C}$ and $51.7^{\circ} \mathrm{C}$, and mean relative humidity between $48.4 \%$ and $84.3 \%$.

Kofidis et al. (2003) found that oregano plants grown in the spring/summer season showed larger glandular hair amounts in their leaves. Assumingly, these structures minimize transpiration and improve oil accumulation in the leaves. The authors emphasized that the oregano essential oil accumulated in the leaves' ventral and dorsal surfaces during the warmest seasons, as well as that all the plants in the experiment conducted during these seasons showed mean content and yield higher than plants grown in the fall/winter season, due to the glandular trichome densification caused by the biomass reduction resulting from water limitation.

Baker and Putievsky (1978) assessed the performance of oregano plants cultivated in the four seasons of the year (in Israel) and, after years of observation, found that the highest essential oil content was shown by cultures held in the spring/summer season. The authors have also pointed out that high temperatures help oregano essential oil biosynthesis. Similar results were found by Trivino and Johnson (2000) in a study conducted in Greece. They found that the meteorological conditions in spring and summer fully meet the oregano cultivation demands and inferred that oregano is a long-day plant.

Tibaldi et al. (2011) analyzed the oregano plant responses to abiotic stress in Carmagnola, Eastern Italy and found that plants grown in days of longer photoperiod show the highest essential oil yield. Circella et al. (1995) assessed the photoperiod effects on oregano essential oil biosynthesis and composition and found the highest essential oil contents in plants grown under photoperiods longer than 13 hours.

The results reported for the Northern hemisphere, as well as in the current study, allow assuming that oregano plants behave as long-day plants in the Southern hemisphere. However, it is necessary conducting further studies to quantify the thermal needs of oregano plants and better understand their seasonal productive performance, since the present study showed that water restriction in the soil throughout oregano plants' phenological cycle favors essential oil biosynthesis.

\section{Conclusions}

The present study shows that water restriction in the soil throughout oregano plants' phenological cycle favors essential oil biosynthesis, whereas, in the pre-flowering stage, it enables higher oregano dry and fresh biomass production. The matric potential $(-60.8 \mathrm{kPa})$ adopted in the irrigation handling throughout the oregano cultivation cycle resulted in the highest mean essential oil content and yield. The matric potential $(-60.8 \mathrm{kPa})$ adopted in the irrigation handling during the oregano pre-flowering stage resulted in the highest mean fresh biomass. The matric potential $(-91.2 \mathrm{kPa})$ adopted in the irrigation handling during the oregano pre-flowering stage resulted in the highest mean dry biomass.

\section{Acknowledgements}

The authors would like to thank the "Walter Accorsi" Medicinal Plant Garden, USP Recicla, the Fruits \& Vegetables Laboratory and the Soils \& Water Quality Laboratory of "Luiz de Queiroz" College of Agriculture for the scientific support, as well as the National Council for Scientific and Technological Development (CNPq-Brazil processes 130853/2014-3 and 161398/2014-6) for granting the scholarships; we also thank the reviewers for their valuable suggestions and comments. 


\section{Conflict of Interests}

The authors declare that there are no conflicts of interest related to this article.

\section{References}

Abdelmajeed NA, Danial EN, Ayad HS (2013). The effect of environmental stress on qualitative and quantitative essential oil of aromatic and medicinal plants. Archives Des Sciences 66:100-120.

Asensio CM, Grosso NR, Juliani HR (2015). Quality characters, chemical composition and biological activities of oregano (Origanum spp.) essential oils from central and southern Argentina. Industrial Crops and Products 63:203-213.

Azizi A, Yan F, Honermeier B (2009) Herbage yield, essential oil content and composition of three oregano (Origanum vulgare L.) populations as affected by soil moisture regimes and nitrogen supply. Industrial Crops and Products 29:554-561.

Bafana A (2013). Diversity and metabolic potential of culturable root-associated bacteria from Origanum vulgare in subHimalayan region. World Journal of Microbiology and Biotechnology 29:63-74.

Baher ZF, Mirza M, Ghorbanli M, Rezaii MB (2002). The influence of waterstress on plant height, herbal and essential oil yield and composition in Satureja hortensis L. Flavour and Fragrance Journal 17:275-277.

Bahreininejad B, Razmjoo J, Mirza M (2013). Influence of water stress on morpho-physiological and phytochemical traits in Thymus daenensis. International Journal of Plant Production 7:151-156.

Basker D, Putievsky E (1978). Seasonal variation in the yields of herb and essential oil in some Labiatae species. Journal of Horticultural Science 53:179-183.

Batista SCO, Carvalho DF, Rocha HS, Thaner Dos Santos H, Medici LO (2013). Production of lettuce automatically watered with a low-cost controller. International Journal of Food, Agriculture and Environment 11:485-489.

Bekhradi F, Luna MC, Delshad M, Jordan MJ, Sotomayor JA, Martínez-Conesa C, Gil MI (2015). Effect of deficit irrigation on the postharvest quality of different genotypes of basil including purple and green Iranian cultivars and a Genovese variety. Postharvest Biology and Technology 100:127-135.

Box GEP, Hunter JS, Hunter WG (2005). Statistics for experimenters: design, innovation, and discovery. WileyInterscience, after Hoboken.

Breusch TS, Pagan AR (1979). A simple test for heteroscedasticity and random coefficient variation. Econometrica 47:1287-1294.

Burmeister J, Guttenberg HV (1960). Die ätherischen öle als produkt eines partiell anaeroben stoffwechsels. Planta Medica 8:1-33.

Busatta C, Mossi AJ, Rodrigues MRA, Cansian RL, Oliveira JV (2007). Evaluation of Origanum vulgare essential oil as antimicrobial agent in sausage. Brazilian Journal of Microbiology 38:610-616.

Chauhan NK, Singh S, Haider SZ, Lohani H (2013). Influence of phenological stages on yield and quality of oregano (Origanum vulgare L.) under the agroclimatic condition of doon valley (Uttarakhand). Indian Journal Pharmaceutical Science 75:89-493.

Chaves MM, Oliveira MM (2004). Mechanisms underlying plant resilience to water deficits: prospects for water-saving agriculture. Journal of Experimental Botany 55:2365-2384.

Chang X, Alderson PG, Wright CJ (2005). Effect of temperature integration on the growth and volatile oil content of basil (Ocimum basilicum L.). Journal of Horticultural Science \& Biotechnology 80:593-598.

Circella G, Franz CH, Novak J, Resch H (1995). Influence of day length and leaf insertion on the composition of marjoram essential oil. Flavour and Fragrance Journal 10:371-374.

Clevenger JF (1928). Apparatus for the determination of volatile oil. Journal of the American Pharmaceutical Association 17:345-349.

Dardioti A, Karousou R, Lanaras T, Kokkni S (2012). Diversity of Satureja pilosa subsp. origanita essential oils: A new “oregano" from East Mediterranean. Biochemical Systematics and Ecology 40:178-183.

Davidenco V, Vega CRC, Piccardi MB, Arguello JA (2015). Development in Origanum ssp.: a simple phenological scale to determine thermal time requirements to critical reproductive events. Scientia Horticulturae 186:70-76. 
Do TKT, Hadji-Minaglou F, Antoniotti S, Fernandez X (2015). Authenticity of essential oils. Trends in Analytical Chemistry 66: 146-157.

Dodd IC, Puértolas J, Huber K, Pérez-Pérez JG, Wright HR, Blackwell MS (2015). The importance of soil drying and re-wetting in crop phytohormonal and nutritional responses to deficit irrigation. Journal of Experimental Botany 66:2239-2252.

Du T, Kang S, Zhang J, Davies WJ (2015). Deficit irrigation and sustainable water- resource strategies in agriculture for China's food security. Journal of Experimental Botany 66:2253-2269.

Economakis CD (1993). Effect of potassium on growth and yield of Origanum dictamnus L. in solution culture. Acta Horticulturae 331:339-344.

El Gendy NA, Leonardi M, Mugnaini L, Bertelloni F, Ebani VV, Nardoni S, ... Pistelli L (2015). Chemical composition and antimicrobial activity of essential oil of wild and cultivated Origanum syriacum plants grown in Sinai, Egypt. Industrial Crops and Products 67:201-207.

EMBRAPA (2013). Sistema brasileiro de classificação de solos. Brasília: Embrapa.

Ekren S, Sonmex C, Ozcakal E, Kurttas YSK, Bayram E, Gurgulu H (2012). The effect of different irrigation water levels on yield and quality characteristics of purple basil (Ocimum basilicum L.). Agricultural Water Management 109:155-161.

Farahani HA, Valadabadi SA, Daneshian J, Shiranirad AH, Khalvati MA (2009). Medicinal and aromatic plants farming under drought conditions. Journal of Horticulture and Forestry 1:86-92.

Farooq M, Wahid A, Kobayashi N, Fujita D, Basra SMA (2009). Plant drought stress: effects, mechanisms and management. Agronomy for Sustainable Development 29:185-212.

Figueiredo AC, Barroso JG, Pedro LG, Scheffer JJC (2008). Factors affecting secondary metabolite production in plants: volatile components and essential oils. Flavour and Fragrance Journal 23:213-226.

Gomes DP, Carvalho DF, Almeida WS, Medici LO, Guerra JGM (2014). Organic carrot-lettuce intercropping using mulch and different irrigation levels. International Journal of Food, Agriculture and Environment 12:323-328.

Guidi L, Landi M (2014) Aromatic Plants: use and nutraceutical properties. In: Gurib-Fakim A (ed) Novel plant bioresources: applications in food, medicine and cosmetics. Wiley, Bognor Regis.

Guvenalp Z, Turan M, Sumer Z (2010). Study on antitumoral activity of some chemical compounds isolated from Origanum vulgare ssp. Planta Medica 76:271.

Hassan FAS, Bazaid S, Ali EF (2013). Effect of deficit irrigation on growth, yield and volatile oil contenton Rosmarinus officinalis L. plant. Journal of Medicinal Plants Studies 1:12-21.

Healy MJR (1956). The analysis of a factorial experiment with additional treatments. The Journal Agricultural Science 47:205-206.

Honermeier B, Ali S, Leschhorn B, Mahmood A, Ijaz M, Russo M, ... Zeller S (2013). Cultivation of medicinal and spice plants in Germany - a review. International Journal of Agriculture \& Biology 15:1379-1388.

Khalil SES, El-Noemani ASA (2015). Effect of bio-fertilizers on growth, yield, water relations, photosynthetic pigments and carbohydrates contents of Origanum vulgare L. plants grown under water stress conditions. AmericanEurasian Journal of Sustainable Agriculture 9:60-73.

Kofidis G, Bosabalidis AM, Moustakas M (2003). Contemporary seasonal and altitudinal variations of leaf structural features in oregano (Origanum vulgare L.). Annals of Botany 92:635-645.

Landi M, Guidi L (2015). Labiateae species in mediterranean environment: unrivaled sources of secondary metabolites. Italus Hortus 22:1-13.

Lemhadri A, Zeggwagh NA, Maghrani M (2004). Anti-hyperglycaemic activity of the aqueous extract of Origanum vulgare growing wild in Tafilalet region. Journal of Ethnopharmacology 92:251-256.

Létourneau G, Caron J, Anderson L, Cormier J (2015). Matric potential-based irrigation management of field-grown strawberry: effects on yield and water use efficiency. Agricultural Water Management 161:102-113.

Lukas B, Schmiderer C, Mitteregger U, Novak J (2010). Arbutin in marjoram and oregano. Food Chemistry 121:185190.

Manukyan A (2011). Effect of growing factors on productivity and quality of lemon catmint, lemon balm and sage under soilless greenhouse production: I. Drought stress. Medicinal and Aromatic Plant Science and Biotechnology 5:119-125.

Matraka M, Ninou E, Giannakoula A, Lazari D, Panou-Filotheou H, Bosabalidisa AM (2010). Effects of soil water content on Mentha spicata L. and Origanum dictamnus L. Israel Journal of Plant Sciences 58:229-239. 
Meneses R, Ocazionez RE, Martinez JR, Stashenko EE (2009). Inhibitory effect of essential oils obtained from plants grown in Colombia on yellow fever virus replication in vitro. Annals of Clinical Microbiology and Antimicrobials 8:1-6.

Mijani S, Eskandri Nasrabadi S, Zarghani H, Ghias Abadi M (2013). Seed germination and early growth responses of hyssop, sweet basil and oregano to temperature levels. Notulae Scientia Biologicae 5:462-467.

Mortensen LM (2014). The effect of air temperature on growth of eight herb species. American Journal of Plant Sciences 5:1542-1546.

Neumann PM (1995). The role of cell wall adjustments in plant resistance to water deficits. Crop Science 35:12581266.

Osakabe Y, Osakabe K, Shinozaki K, Tran LSP (2014). Response of plants to water stress. Frontiers in Plant Science 5:1-8.

Paraskevakis N (2015). Effects of dietary dried Greek Oregano (Origanum vulgare ssp. hirtum) supplementation on blood and milk enzymatic antioxidant indices, on milk total antioxidant capacity and on productivity in goats. Animal Feed Science and Technology 209:90-97.

Penka M (1978). Influence of irrigation on the contents of effective substances in officinal plants. Acta Horticulturae 73:181-198.

R Development Core Team (2013). A Language and environment for statistical computing. Austria: R Foundation for Statistical Computing.

Raut S, Karuppayil SM (2014). A status review on the medicinal properties of essential oils. Industrial Crops and Products 62:250-264.

Rioba NB, Itulya FM, Said M, Dudai N, Bernstein N (2015). Effects of nitrogen, phosphorus and irrigation frequencyon essential oil content and composition of sage (Salvia officinalis L.). Journal of Applied Research on Medicinal and Aromatic Plants 2:21-29.

Said-Al Ahl H, Omer EA, Naguib NY (2009). Effect of water stress and nitrogen fertilizer on herb and essential oil of oregano. International Agrophysics 23:269-275.

Sangwan NS, FaroogI AHA, Shabid F, Sangwan RS (2001). Regulation of essential oil production in plants. Plant Growth Regulation 34: 3-21.

Savini I, Arnone R, Catani MV, Avigliano L (2009). Origanum vulgare induces apoptosis in human colon cancer CaCo2 cells. Nutrition and Cancer 61:381-389.

Seghatoleslami M, Feizi H, Mousavi G, Berahmand A (2015). Effect of magnetic field and silver nanoparticles on yield and water use effi ciency of Carum copticum under water stress conditions. Polish Journal of Chemical Technology 17:110-114.

Shao HB, Chu LY, Jaleel CA, Zhao CX (2008). Water-deficit stress-induced anatomical changes in higher plants. Comptes Rendus Biologies 331:215-225.

Sivakumar D, Bautista-Bãnos S (2014). A review on the use of essential oils for postharvest decay control and maintenance of fruit quality during storage. Crop Protection 64:27-37.

Soil Survey Staff (1999). Soil taxonomy: a basic system of soil classification for making and interpreting soils surveys. Department of Agriculture. Soil Survey Division. Soil Conservation Service, Washington

Souza EL, Stamford TLM, Lima EO (2006). Sensitivity of spoiling and pathogen- food related bacteria to Origanum vulgare L. (Lamiaceae) essential oil. Brazilian Journal of Microbiology 37:527-532.

Telci I, Hisil Y (2008). Biomass yield and herb essential oil characters at different harvest stages of spring and autumn sown Coriandrum sativum. European Journal Horticultural Science 73:267-272.

Tibaldi G, Fontana E, Nicola S (2011). Growing conditions and postharvest management can affect the essential oil of Origanum vulgare L. ssp. hirtum (Link) Ietswaart. Industrial Crops and Products 34:1516-1522.

Trivellini A, Lucchesini M, Maggini R, Mosadegh H, Villamarin TS, Vernieri P, ... Pardossi A (2016). Lamiaceae phenols as multifaceted compounds: bioactivity, industrialprospects and role of "positive-stress". Industrial Crops and Products 83:241-254.

Trivino MG, Johnson CB (2000). Season has a major effect on the essential oil yield response to nutrient supply in Origanum majorana. Journal of Horticultural Science \& Biotechnology 75:520-527.

Torres LE, Brunetti PC, Baglio C, Bauza PG, Chaves AG, Massuh Y, ... Ojeda MS (2012). Field evaluation of twelve clones of oregano grown in the main production areas of Argentina: identification of quantitative trait with the highest discriminant value. International Scholarly Research Network Agronomy 2012:1-10. 
Uhland RE (1951). Rapid method for determining soil moisture. Soil Science Society Proceedings 15:391-393.

Van Genuchten MTA (1980). A closed form equation for predicting the hydraulic conductivity of unsaturated soils. Soil Science Society of America Journal 44:892-898.

Voltolina G (2017). Piante Officinali Dalla coltivazione alla vendita nell'azienda agrícola multifunzionale Manuale agroerboristico. Verona: Edizioni L'Informatore Agrario.

Whalley WR, Ober ES, Jenkins M (2013). Measurement of the matric potential of soil water in the rhizosphere. Journal of Experimental Botany 64:3951-3963.

Xu DH, Li JH, Fang XW, Wang G (2007). Changes in soil water content in the rhizosphere of Artemisia ordosica: evidence for hydraulic lift. Journal of Arid Environments 69:545-553.
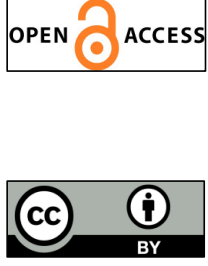

The journal offers free, immediate, and unrestricted access to peer-reviewed research and scholarly work, due SHST supports to increase the visibility, accessibility and reputation of the researchers, regardless of geography and their budgets. Users are allowed to read, download, copy, distribute, print, search, or link to the full texts of the articles, or use them for any other lawful purpose, without asking prior permission from the publisher or the author.

License - Articles published in Notulae Scientia Biologicae are Open-Access, distributed under the terms and conditions of the Creative Commons Attribution (CC BY 4.0) License.

(c) Articles by the authors; SHST, Cluj-Napoca, Romania. The journal allows the author(s) to hold the copyright/to retain publishing rights without restriction. 